\title{
HIV/AIDS Stigma Reduction as a Risk Factor for New HIV Infections: A Lived Experience Study of HIV Positive Young Women in a Rural Setting of Northern Uganda
}

Amir Kabunga ( $\sim$ amirkabs2017@gmail.com )

Lira University https://orcid.org/0000-0002-0481-2220

Maxson Anyolitho Kenneth

Mira University

Research

Keywords: Coping, HIV, Stigma, Uganda, Young women

Posted Date: November 5th, 2020

DOI: https://doi.org/10.21203/rs.3.rs-101843/v1

License: (c) (1) This work is licensed under a Creative Commons Attribution 4.0 International License. Read Full License 


\section{Abstract}

\section{Background}

HIV/AIDS-related stigma is a global problem among HIV patients with far-reaching implications. However, stigma reduction among HIV positive young women may be a catalyst to new HIV infections. This study aimed at exploring the coping strategies used and influence of HIV-related stigma on new infections among HIV positive young women in a rural setting.

\section{Methods}

A cross-sectional exploratory research design was used. HIV positive young women in the catchment area of Aboke Health Center IV were part of the study. In-depth interviews were used to collect data and analysis was done using thematic approach.

\section{Results}

The results from the data showed that acceptance of counseling and social support are some of the ways HIV-positive young women coped up with stigma. The results also showed that While HIV-related reduction has influenced positively for some HIV positive young mothers, to others as HIV-related stigma reduces, young women living with HIV are likely to get involved in risky sexual activities like having unprotected sex thus increasing HIV transmission.

\section{Conclusions}

HIV-related reduction has influenced positively for some HIV positive young mothers. However, to others as HIV-related stigma reduces, young women living with HIV are likely to get involved in risky sexual activities like having unprotected sex thus increasing HIV transmission. Based on the results, there is need to understand the negative effects HIV-related stigma reduction as this may have implications for further transmission and control of HIV pandemic. Also, further research is needed to understand whether lessening HIV-related stigma encourages risky sexual behaviors among young women.

\section{Introduction}

Globally, an estimated 1.8 million young people are living with HIV with $30 \%$ of all new infections occurring among the young men and women aged 15-24 years [1]. New HIV infections continue in some regions and among key populations[1]. In 2017, the Sub-Saharan Africa region accounted for $25 \%$ of the global burden of new infections among young women and adolescents yet it accounted for $10 \%$ of the global population [2], [3]. Statistics also show that weekly, at least 6000 adolescent girls and young women become infected with HIV [4]. In eastern Africa, gender disparity in HIV infections is three times higher among young women than among young men [1]. To achieve an HIV/AIDS free society there is need to understand and reduce the burden of new infection in young women [5]. 
Between 2010 and 2018, a record-breaking numbers of people accessed Antiretroviral Therapy (ART) becoming virally suppressed, resulting in a $33 \%$ decline in the number of people dying from an AIDSrelated illness [2]. The increased ART uptake is attributed to among others, HIV/AIDS stigma reduction efforts [6]. HIV positive young women have tried to overcome HIV-related stigma through acceptance, social support and counseling [7], [8]. However, there is limited information on how young women in rural setting cope with stigma [8]. Besides, despite the achievements, a significant HIV burden still remains with 38 million people living with HIV and an estimated 1.7 million individuals worldwide diagnosed with new HIV infections in 2018 and young women are disproportionately affected [9]. Significant drivers of the upsurge of new infections in this key population include gender disparity, violence against women and relationship with older men [10]. However, it is still not clear how stigma reduction influences new HIV infections.

Uganda has made a significant progress in HIV epidemic response for the last three decades: preventing newly infected cases, reducing HIV-related mortality and fatality [11]. HIV related stigma was equally tackled to increase ART uptake with great success [12]. However, these achievements are under threat from new HIV infections. Currently Uganda has about 170,000 young men and women living with HIV and the figures are likely to increase because more youth remain susceptible to new infections [13]. A third of these infections are mostly registered among young women aged 15-24 [14]. Reduction in HIV- related stigma which has for long been cited as a major barrier to accessing prevention, care and treatment services [11], may now be a contributing factor to new HIV infections. However, there seems to be limited literature to support this assertion. This study attempts to fill the gap by exploring whether reduction in HIV-related stigma influences HIV transmission among young women especially in the rural setting.

\section{Methods}

The study setting was Aboke health center IV, Kole district, northern Uganda which is located approximately 30 kilometers to the West of Lira City. A cross-sectional exploratory research design was employed to explore coping strategies and influence of stigma reduction on new HIV infections among young women attending outpatient Aboke health center IV. The period of data collection was August 2020 .

\section{Participants}

Study participants were HIV-positive young women aged between 15-24 years old within the catchment area of Aboke Health Center IV, Kole District. A total of 15 HIV positive young women attending Aboke health center IV participated in the study. The sample size was estimated using saturation principle as applied in qualitative study [15]. Participants were purposively selected to get those who were capable of giving a richer narrative of their stigma experiences, coping strategies and how stigma reduction influences HIV infections. The inclusion criteria were HIV positive young women aged between 15-24 years of age, diagnosed with HIV and receiving ART.

\section{Instrument}


Data was collected using an interview guide comprising of three main sections; socio-demographic characteristics, HIV-related stigma coping strategies and how stigma reduction influences HIV infections among young women. The researcher developed the interview guide using guidelines provided by Huberman and Miles [16]. Also the development of interview guide was based on literature on stigma and coping strategies with input from experts who had experiences in HIV-related stigma. The guide was translated and back translated to establish consistency.

\section{Procedure}

Lira university graduates trained in micro-research and fluent in both English and Langi conducted faceto-face in-depth interviews in a private setting. The interviews lasted for 25-30 minutes and responses from the participants were recorded using a voice recorder. Participants were asked to express how they coped with stigma and how stigma reduction influences their sexual activities. They were approached on clinic days after permission was granted by District Health Officer of Kole District. Interviewers continuously probed and watched the body language of respondents to elicit rich and accurate perspectives on HIV-related stigma reduction experiences and how it influences their sexual activities.

\section{Data analysis}

Audio recording of the interviews were transcribed and later translated into English. To assess the quality and dependability of transcripts two separate experts reviewed the translation. A seven phase data analysis framework described by Braun and Clarke [17] was used. After reading and transcription of recorded data into written document, data was coded which enabled the researchers to identify categories and patterns within data. Different colors were used to distinguish between themes.

Thereafter, themes were identified, reviewed and named. Lastly synthesis of the themes, analysis of data and interpretation of results followed to provide an understanding of stigma reduction and its influence on HIV Infections among young women.

\section{Results}

Twenty five participants were eligible for the study. However, in-depth interviews were conducted with 15 HIV positive young women. Participants' age ranged from 17-24 years. All the participants were Langi by tribe, seven were married, five were single and three were divorced.

\section{Prevalence of Stigma after Testing HIV Positive}

HIV-related stigma as a phenomenon was considered to be a major barrier to reaching those who are at risk or infected with HIV/AIDS. Although HIV-related stigma is a general phenomenon which affects people living with HIV/AIDS, its reduction in this paper was presumed to precipitate new HIV infections among young women in Kole district in Uganda.

Participants narrated feelings of stigma associated with HIV infections which highlighted negative public view of the disease. Some of the participants' narratives reflect instances of experiencing severe stigma 
when they first tested with HIV. This is because the disease is associated with promiscuity. Young women diagnosed with HIV had a fear of being found out because this would lead to community rejection. For some of the participants, the situation was overwhelming, challenging and grappled with stigma. Hence, they were faced with the dilemma of either disclosing the status or not. Based on the interviews a 22 year old participant narrated her predicament as follows:

"At first I did not believe I could disclose my status because I thought that my people would think I was infected as a result of prostitution. But that is what they think any way, they would call me immoral....... But after receiving counseling services, I disclosed to my mother, my brother and later the friends. I believe everyone in the village now knows my status".

Another 20 year old said;

"The first few weeks or months when I tested positive, I believed that everyone knew I had the disease. I literally reminded in the house, I did not want see anybody including the closest friend. I felt rejected by all people. But with time I liked myself and I was very happy. My husband who is HIV-negative supported me in this process of self-acceptance. In fact I realized that HIV is another disease".

\section{HIV-related stigma coping strategies used by young women living with HIV}

Participants' narratives show that for some time after the diagnosis, most young women perceived HIV as "death sentence" and thus no hope of survival. However with counseling services and social support the situation lessened. They felt better with more positive outlook and coped better with HIV-related stigma. Hence it can be argued that although the HIV positive young women are challenged with stigma, they were determined to overcome it and live a normal life. In this regard, a 21 year old HIV positive young woman expressed:

“..... I don't think anyone should blame me for being HIV positive because I don't know where this disease came from. I now live my life and don't care what others are saying.... they can go to hell, for all I care about is my life".

Many participants suggested that counseling was done thoroughly by trained counselors. Intense counseling therefore was vital in overcoming HIV-related stigma. A 24 old positive young woman recounted how she overcame stigma through the assistance of friends and professional counselors;

"Trust me I have outgrown stigma and I can see a bright future. That stress ended with help of friends and counselors"

Accepting HIV status was an important method to cope and deal with stigma. One respondent underscored the importance of self-acceptance;

"... I realized I need to help myself or else I would die very fast. I simply accepted my status, live for my child and loved ones" 
Amidst reports of stigma, family support proved to be invaluable for other young girls living with HIV. The participants' narratives show that positive support extended to them by family members, health professional and friends is valuable in dealing with stigma. Consequently they overcame the HIV-related stigma as an 18 year old young woman said;

"My people helped me so much and perhaps that's why am still alive and healthy. They supported me morally, materially or otherwise. My friends told me never to mind what other people are saying, after all am not alone and am not going to die soon"

\section{The influence of HIV-related stigma reduction on new infections among young women}

Explicitly, participants overcame the issue of stigma and seem to be more open to life. For these participants, this is a plus but there is now a worry that a 'normal life' may be causing new HIV infections among young women. Some participants even reached a point of believing that because the community is no longer ostracizing them, they are not HIV positive and can have sex without any guilt of transmission. Some young women reported that they pushed HIV problems out of their minds and believe that they are HIV negative and could not transmit the disease to others. One young woman aged 20 year old put it this way;

"Am I HIV positive? Yes I was tested positive but I don't believe that I have the disease... I live my normal life, I enjoy sex normally.... after all am still a young and attractive lady. Am a good looking woman, I began having sex, this is the truth, I have a partner from the neighborhood and he doesn't care about my status".

Some participants agreed that people in the community do not treat them differently. They freely associate and as a result they can have unprotected sex as a 24 year young woman said;

"Nothing is going to happen if I have unprotected sex, I have sex regularly and am not worried about the future. There are some old notches I need to settle first, and then I can die"

Other participants recounted that they know they have the disease but they need to have fun just like others in the same age bracket as respondent aged 18 years old said;

"Yes am infected, but that status is not going change, not now, not ever. I have to enjoy life just like my age mates. They have boyfriends, others have many boyfriends and others are happily married thus enjoying what "nature" provided. I need to be happy for as long as men like me".

Yet still another 21 year old said;

"I realized that having sex was the way though at first I was scared .... but at the same time, I felt that I need to have fun. I decided to move on and follow my desires regardless of the consequences. I don't need stress anymore; I need a normal life too". 
Some participants even decided to get involved in risky sexual activities like having unprotected sex thus increasing HIV transmission. One respondent aged 23 years old said;

"...... this is not a big deal, many men who originally did not want to come near me or associate with me are now so close, I mean very close. I think that they (men) think it is just a disease and nothing more. I feel the same too, what else, I live with this condition, those who get sick, they will get medication just like $m e^{\prime \prime}$.

Overcoming HIV-related stigma however impacted HIV positive pregnant mothers positively. Somme participants after overcoming the issues related to stigma they did not only initiate the process of acceptance of their HIV status but also lived a responsible life. They understood the importance of taking medicine in time and not engaging in unsafe sexual activities. A 23 year old said;

"Before I received professional counseling, I was very sick and I felt rejected by the community. But when my I consulted my counselor, I felt very good and strong. I realized that I had to live responsibly; I needed to live longer and also protect my loved ones from getting infected".

Other participants also narrated that they enjoy sex but they use condoms. Some participants also narrated that they used their own protection if men disagreed. They attributed this to health education and counseling.

"When I was diagnosed with HIV, I wanted to have with everyone and did not want to take ARV. But this changed when I received counseling and heard some good and educative information over the radio. I can't afford to infect others as I will be destroying myself too. I carry with me condoms and I make sure they are used and used properly".

\section{Discussion}

New HIV infections continue to rise in the world and among key populations including young men and women aged 15-24 years with young women being disproportionately affected [12]. This study highlights positive coping strategies used by HIV positive young women in a rural setting to reduce the effects of HIV-related stigma and how reductions in HIV related stigma influences new HIV transmission. In sum, the common stigma coping strategies used by HIV positive young mothers were acceptance, social support and counseling. Acceptance of HIV status aids individuals to positively change their situation. Besides, the supportive environment implied that the young women living with HIV lived a positive life and adhered to medical schedules [8]. Therefore efforts towards instilling positive attitudes towards people living with HIV in the community may lead to acceptance of these people and thus receive the required support [18]. Findings from this study support existing literature [7], [8].

Stigma is a serious burden for individuals living with HIV/AIDS as it may prevent them from seeking medical care [7] and contributes to less interactions with peers, friends, family and community members [12]. Therefore reducing HIV-related stigma is an important step in improving the quality of life of young 
women living with HIV. A number of programs to reduce HIV-related stigma such as media campaign, education and others have been launched and they have been effective in Uganda [6]. Also a review of other literature shows that these approaches result in changes in expressed attitude towards people living with HIV [19]. Paradoxically, it is possible that the reduction in HIV-related stigma may be a catalyst for HIV new infections among young women. Results in this study demonstrate that reduction in HIV-related stigma increases risky sexual behaviors thus increasing the spread of the disease. It seems that stigma reduction contributes to psychological and social or psychosocial adjustments among young women living with HIV. The perceived threats of HIV and HIV-related stigma have lessened with increased treatment and campaign against stigma. It is also important to note that reduced stigma seem to empower young women to discuss their sexual desires. There is increase in optimism and the belief that HIV treatment eliminates the risk of HIV transmission [20]. The findings indicate that as HIV-related stigma lessens many have renewed sexual desires. Unfortunately, some engage in unprotected sex thus increasing the transmission of the disease.

The study limitations included the use of a cross-sectional design, inclusion of only positive young women from a single outpatient health center in a rural setting. The results may not be generalized to HIV positive young men and old women. Mixed research studies may contribute to better understanding of the topic. Future research should include all categories of people living with HIV for generalization of results.

\section{Conclusion}

Reduction in HIV related stigma is crucial in promoting positive living among young people. However, there are now concerns that lessening this HIV-related stigma is associated with increased risky sexual behaviors especially among young women. While HIV-related reduction has influenced positively for some HIV positive young mothers, to others as HIV-related stigma reduces, young women living with HIV are likely to get involved in risky sexual activities like having unprotected sex thus increasing HIV transmission. There is therefore the need to understand the negative effects of HIV-related stigma reduction as this may have far reaching implications for the transmission and control of HIV pandemic. Also, further research is needed to understand whether lessening HIV-related stigma encourages risky sexual behaviors among young women.

\section{Declarations}

\section{Ethics approval and consent to participate}

Ethical clearance was granted by institutional review board of Gulu University (GUREC-047-20). A written informed consent was obtained from all the participants before data collection. Privacy and confidentiality were maintained during the entire process of data collection and analysis. Interviews were audio-taped with participants' permission. Standard operating procedures of COVID-19 prevention were maintained including observance of social distancing and wearing of face masks. 


\section{Consent for publication}

This manuscript doesn't any personal data of the participants

\section{Availability of data and material}

This data set is part of a bigger study. However the data sets analyzed is available from the corresponding author on realistic request.

\section{Competing interests}

There is no competing interest

\section{Funding}

The author(s) received no financial support for the research, authorship, and/or publication of this article

\section{Authors' contributions}

AK conceptualized the study and was involved in the data collection, analysis, and drafting of the manuscript. MAK participated in proposal writing, analysis, and drafting of the manuscript. All authors read and approved the final manuscript.

\section{Acknowledgment}

We acknowledge all those who accepted to participate in this study. We also acknowledge the graduate students of Lira University for data collection.

\section{Authors' information}

${ }^{1}$ Is a lecturer and psychologist in the Department of Psychiatry, ${ }^{2}$ Department of Public Health, Mira University, P.O. Box 1035, Lira, Uganda

\section{References}

[1] S. S. A. Karim and C. Baxter, 'HIV incidence rates in adolescent girls and young women in sub-Saharan Africa', Lancet Glob. Heal., vol. 7, no. 11, pp. e1470-e1471, 2019, doi: 10.1016/S2214-109X(19)30404-8.

[2] J. U. N. P. on H. (UNAIDS), 'Global HIV \& AIDS statistics-2018 fact sheet', UNAIDS. org (http//www. unaids. org/en/resources/fact-sheet)[Accessed August 8, 2018] Export Cit., 2018.

[3] M. Mabaso, Z. Sokhela, N. Mohlabane, B. Chibi, K. Zuma, and L. Simbayi, 'Determinants of HIV infection among adolescent girls and young women aged 15-24 years in South Africa: A 2012 populationbased national household survey', BMC Public Health, vol. 18, no. 1, pp. 1-7, 2018, doi: 10.1186/s12889018-5051-3. 
[4] UNHCR, 'Uganda Country Refugee Response Plan', UN Refug. Agency, no. Jan 2019-Dec 2020, p. 72, 2019, [Online]. Available: http://reporting.unhcr.org/sites/default/files/Uganda Country RRP 2019-20 \%28January 2019\%29.pdf.

[5] Q. A. Karim and R. Dellar, 'Inclusion of adolescent girls in HIV prevention research-an imperative for an AIDS-free generation', J. Int. AIDS Soc., vol. 17, no. 1, 2014.

[6] A. C. Tsai et al., 'Evidence from a cohort study in rural Uganda', AIDS Behav., vol. 17, no. 8, pp. 27252731, 2013, doi: 10.1007/s10461-013-0503-3.How.

[7] M. Kotzé, M. Visser, J. Makin, K. Sikkema, and B. Forsyth, 'The coping strategies used over a two-year period by HIV-positive women who had been diagnosed during pregnancy', AIDS Care, vol. 25, no. 6, pp. 695-701, 2013.

[8] S. Ashaba et al., 'Understanding coping strategies during pregnancy and the postpartum period: a qualitative study of women living with HIV in rural Uganda', BMC Pregnancy Childbirth, vol. 17, no. 1, pp. $1-10,2017$.

[9] S. Harrison and X. Li, 'Toward an enhanced understanding of psychological resilience for HIV youth populations', AIDS Care, vol. 30, no. sup4, pp. 1-4, 2018.

[10] Q. A. Karim, S. Sibeko, and C. Baxter, 'Preventing HIV infection in women: a global health imperative', Clin. Infect. Dis., vol. 50, no. Supplement_3, pp. S122-S129, 2010.

[11] P. North, 'Communities at the heart?', Urban renaissance?, pp. 121-138, 2017, doi: 10.2307/j.ctt1t898kc.12.

[12] Avert, 'HIV stigma and discrimination', 2019.

[13] A. C. Schuyler et al., 'Mobility among youth in Rakai, Uganda: Trends, characteristics, and associations with behavioural risk factors for HIV', Glob. Public Health, vol. 12, no. 8, pp. 1033-1050, 2017.

[14] UNAIDS, 'HIV prevention among adolescent girls and young women', Geneva, 2016.

[15] B. Saunders et al., 'Saturation in qualitative research: exploring its conceptualization and operationalization', Qual. Quant., vol. 52, no. 4, pp. 1893-1907, 2018.

[16] A. M. Huberman and M. B. Miles, 'Narrative analysis', Qual. Res. companion. Thousand Oaks, CA Sage Publ., 2002.

[17] V. Braun and V. Clarke, Successful qualitative research: A practical guide for beginners. sage, 2013.

[18] L. Brown, K. Macintyre, and L. Trujillo, 'Interventions to reduce HIV/AIDS stigma: what have we learned?', AIDS Educ. Prev., vol. 15, no. 1, pp. 49-69, 2003. 
[19] A. MacHowska, B. L. Bamboria, C. Bercan, and M. Sharma, 'Impact of HIV-related stigma-reduction workshops' on knowledge and attitude of healthcare providers and students in Central India: A pre-test and post-test intervention study', BMJ Open, vol. 10, no. 4, pp. 1-10, 2020, doi: 10.1136/bmjopen-2019033612.

[20] C. Kennedy, K. O'reilly, A. Medley, and M. Sweat, 'The impact of HIV treatment on risk behaviour in developing countries: a systematic review', AIDS Care, vol. 19, no. 6, pp. 707-720, 2007. 